\title{
El aprovechamiento de los recursos naturales en el Parque Natural Bahía de Cádiz
}

José Manuel López Vázquez

Biólogo. Asesor Técnico del Parque Natural Bahía de Cádiz

\begin{abstract}
Resumen
La ancestral intervención humana ha determinado en gran modo el actual paisaje de la Bahía de Cádiz. Salinas, pesca, marisqueo, acuicultura y un rico patrimonio natural de alto valor biológico, estético y recreativo caracterizan a este espacio, intensamente humanizado, situado a caballo entre la tierra y el mar.
\end{abstract}

\section{Palabras clave}

Cádiz / Marismas / Recursos naturales / Salinas / Pesca / Marisqueo / Acuicultura

\section{Las Salinas de la Ribera Gaditana}

Las salinas configuran la imagen predominante de la Bahía de Cádiz y por el papel que llegaron a representar en la estructura productiva de la comarca han constituido el símbolo indiscutible de esta durante siglos.

Son las salinas paisajes antrópicos, transformados por el hombre, ya que aun partiendo de un medio físico de especiales características es necesario preparar el terreno, construir la salina propiamente dicha, para poder extraer ese valioso recurso que para el hombre, especialmente en el pasado, ha constituido la sal.

Las marismas son muy extensas en esta región y presentan una distribución de caños cuya funcionalidad, unida a la alta insolación, escasas precipitaciones y continuos vientos favorecedores de la evaporación, hacen que la Bahía gaditana sea un lugar óptimo para la construcción de salinas.

Aunque se tienen referencias de aprovechamientos salineros desde el Neolítico, en las marismas gaditanas estas se desarrollaron de forma notable a partir del establecimiento de los fenicios y más tarde de los romanos (9), quienes las aprovecharon como base para la implantación de una notable industria de

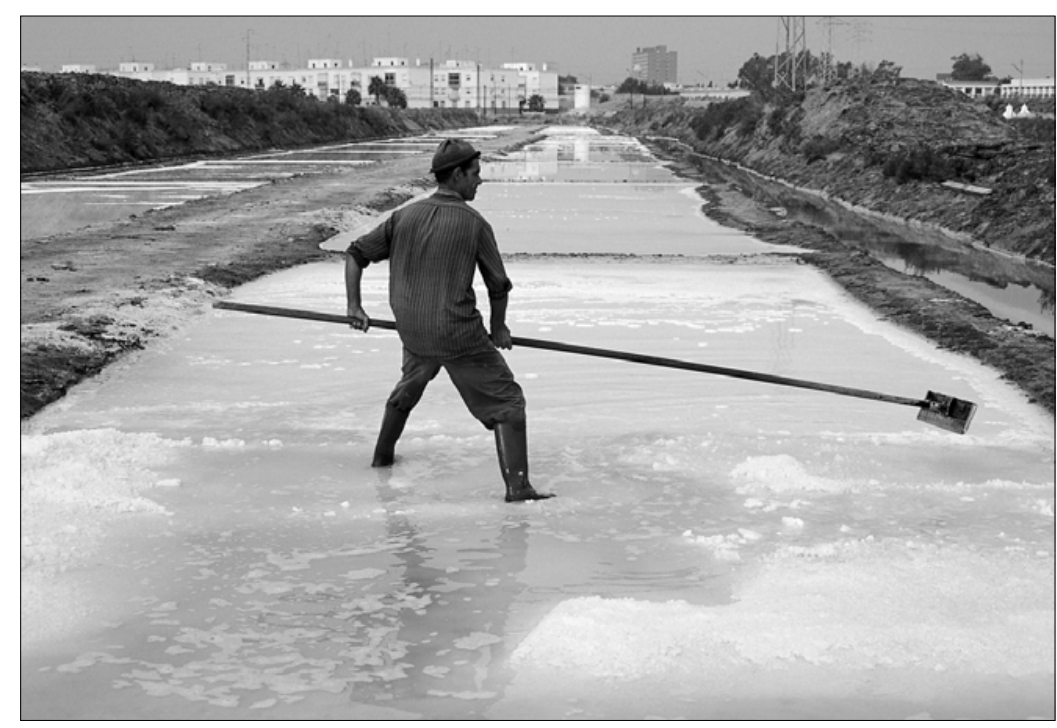

salazón de pescado. Con la caída del imperio romano las salinas gaditanas caen en una crisis que no fue superada hasta el siglo XIII cuando al pasar a manos de nobles, instituciones eclesiásticas y municipios adquieren un nuevo auge para volver a decaer durante los siglos XVII y XVIII debido a los fuertes impuestos exigidos por la corona. La época de esplendor de las salinas gaditanas llega con el siglo XIX pasándose de 66 salinas en 1823 a las 152 que llegaron a existir a principios del siglo $X X$ ocupando una superficie de algo más de 5.500 Has. y una producción anual en torno a las 300.000 toneladas. Desde entonces y bien entrado el siglo $X X$, debido a la fuerte baja del precio de la sal en los mercados internacionales y a la inadaptación de esta industria a las mejoras tecnológicas, se inicia una etapa de decadencia que perdura en la actualidad quedando solo en activo una decena de salinas que producen aproximadamente 37.000 toneladas/año, subsistiendo en claras condiciones de precariedad y habiendo sido el resto abandonadas, rellenadas o transformadas para la acuicultura. Hoy día tan solo algunas grandes salinas de construcción más reciente y marcado carácter industrial, muy alejado de la vieja tradición salinera, parece que pueden tener un rendimiento económico que garantice la continuidad de este aprovechamiento en el futuro.

A primera vista extraños laberintos de muros y estanques sin aparente sentido, las salinas tradicionales son sin embargo magníficas obras de ingeniería, adaptadas al entorno y perfectamente diseñadas para el fin con que fueron concebidas. Los elementos principales de los que se compone una salina tradicional son: Vuelta de afuera, muro externo que sepa-
Labores de extracción de sal en la tajería de una salina tradicional. Foto: Antón Ramírez 
ra la salina del caño natural que la alimenta de agua. Estero, primer depósito de grandes dimensiones en que se almacena el agua que con la subida de la marea penetra desde el caño de alimentación a través de la compuerta de marea y sirviendo de decantador de los sólidos en suspensión. Lucios, y vueltas de periquillo y de retenida, comunicados entre sí por los denominadas largaderos y que no son más que depósitos delimitados por muros en forma de largos y sinuosos zigzag de cada vez menor profundidad en que el agua lentamente se va evaporando actuando así como concentradores de la sal. Tajería, conjunto de pequeños depósitos, tajos, de muy escasa profundidad y dispuestos en cuadrículas donde la sal finalmente cristaliza y es extraída para ser acumulada en montones que luego serán transportados a los saleros o depósitos que cada salina tenía en las proximidades de su embarcadero o carretera. Como complemento a estas estructuras la Casa salinera, producto de la arquitectura popular, de humilde tamaño y construida con materiales y medios autóctonos, servía de vivienda, taller, almacén, pajar, cuadra y comedor o albergue ocasional de los temporeros.

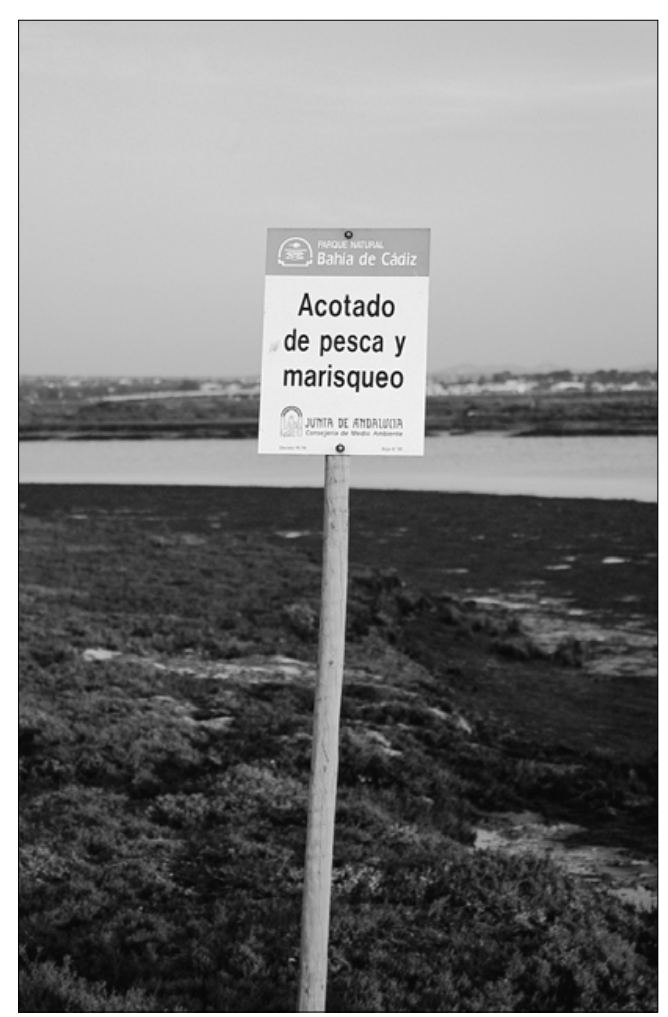

Tantos siglos de influencia de cultura salinera y su particular modo de vida, la amplia extensión de territorio que ha llegado a ocupar, su singular compartimentación espacial, sus características edificaciones y estructuras, han terminado por perfilar el actual paisaje de la Bahía, condicionando el resto de usos del territorio y dotado de una marcada impronta a este territorio, constituyéndose así en un rico patrimonio arquitectónico y cultural que corre el riesgo de desaparecer a muy corto plazo si desde las administraciones no se adoptan medidas urgentes para su conservación.

\section{La pesca y el marisqueo}

Merced a las especiales características fisico-químicas e hidrodinámicas de la Bahía de Cádiz y su entorno los caños de sus marismas son una importante área de desove y cría para numerosas especies de peces, moluscos y crustáceos marinos, muchas de ellas de gran interés comercial (I), lo que ha propiciado la existencia ya desde tiempos prehistóricos de un intensivo aprovechamiento de estos recursos por parte del hombre.

Las primeras evidencias de este aprovechamiento las hallamos en el yacimiento arqueológico de El Estanquillo, en el municipio de San Fernando, en el que se ha detectado una abundante presencia de restos de peces y moluscos que datan del paleolítico inferior y épocas posteriores. Ya en época fenicia y cartaginesa pero sobre todo en el período de dominación romana se hicieron muy famosos en todo el imperio alimentos procedentes de esta región y derivados del pescado como el garum y diversas salazones (9). Desde entonces hasta nuestros días la explotación pesquera y marisquera en la bahía, aunque con altibajos, ha venido manteniendo siempre un elevado protagonismo en la comarca constituyéndose en una de las principales fuentes de ingresos y dejando una fuerte huella en el carácter y cultura de sus gentes.

En los últimos años, fruto de la crisis sufrida por el sector de las pesquerías por la reducción de sus cupos de capturas en los caladeros internacionales y del bajo nivel de empleo en la comarca, se ha propiciado un aumento considerable de las personas que en el entorno de la Bahía ejercen de forma profesional o semiprofesional actividades en este sector extractivo. Ello unido a la permisividad o falta de eficacia de las autoridades competentes en la materia ha dado lugar a un incremento espectacular de la pesca y el marisqueo ilegal en la Bahía, estando actualmente sus recursos sometidos a una grave sobreexplotación, lo cual queda claramente demostrado por el hecho de que cada año ha de aumentarse el esfuerzo para obtener igual volumen de capturas ( I0).

Se sabe que en la actualidad la inmensa mayoría de las capturas realizadas en el interior de la Bahía se ejercen y comercializan al margen de la norma legal, estando oficialmente reconocidas y legalizadas para esta actividad tan solo unas 30 embarcaciones y 60 tripulantes de las más de 1.400 embarcaciones y aproximadamente 1.800 tripulantes que en 1996 faenaban en las aguas interiores y caños de la Bahía. Ello en unión del aproximadamente medio millar de mariscadores que, sin licencia, accederían desde tierra hasta las zonas intermareales para efectuar sus capturas nos da idea clara de la ilegalidad en que se mueve este sector productivo $(10)$.

Las capturas de pesca y marisqueo se comercializan fundamentalmente para su consumo humano en mercados, restaurantes y establecimientos de hostelería de la propia Bahía y tan solo parece existir un buen volumen de comercialización hacia el exterior 
en el caso de las especies capturadas como cebos para la pesca deportiva, principalmente gusanos poliquetos y algunos crustáceos.

Las artes de captura más utilizadas en aguas de la Bahía son los trasmallos, redes de enmalle y palangres para la pesca y los rastros mecánicos y herramientas manuales para el marisqueo. La mayoría de las embarcaciones utilizadas son de pequeño calado y dimensiones, generalmente de tipo patera con motor fueraborda, contando normalmente con solo uno o dos o tres tripulantes.

Las principales especies objeto de pesca en el interior del Parque Natural Bahía de Cádiz son, ordenadas por de mayor a menor porcentaje de capturas, las siguientes: lisas (Liza aurata, L. ramada, L. saliens, Mugil cephalus, Chelon labrosus) 17\%, pez sapo (Halobatrachus didactylus) $13 \%$, mojarras y sargos (Diplodus annularis, D. belloti, D. vulgaris, D. sargus, D. puntazzo, D. cervinus) 12\%, dorada (Sparus aurata) $9 \%$, lenguados (Solea vulgaris, S. senegalensis) $8 \%$, robalos o lubinas (Dicentrarchus labrax, D. punctatus) $6 \%$, herrera (Lithognatus mormyrus) 5,5\%, salema (Sarpa salpa) 5\%, corvina (Argyrosomus reginus) $4 \%$, pejerrey (Aterina boyeri) 3,5\%, anguila (Anguilla anguiIla) 2,3\%, y en cantidades menores otras especies como la piraña (Fundulus heteroclitus), pargo (Pagrus pagrus), congrio (Conger conger), cazón (Galeorthinus galeo), aguja (Belone belone), garapello (Pagellus beIlotti), breca (Pagellus erythrynus), hurta (Pagrus caeruleosticctus), jurel (Trachurus trachurus) o el perrillo (Pomatoschistus microps). Como idea del volumen ilegal de capturas de estas especies diremos que en el período de enero de 1995 a junio de 1996 se calcularon, en 10 asentamientos de pesqueros ilegales que faenan en el interior del Parque Natural, alrededor de 616 toneladas de capturas de pescado de 38 especies diferentes.

En cuanto a los moluscos para el mismo período y asentamientos se estimó en aproximadamente 61 toneladas de 18 especies el volumen de las captura, siendo el choco (Sepia officinalis) la más abundante con el 17\%, seguido del pulpo (Octopus vulgaris) 15\%, coquina de arena (Donax trunculus), coquina de fango (Scrobicularia plana) y navaja o muergo (Solem marginatus) con el 10\% cada una de ellas, burgaillo (Manodonta turbinata), cañailla (Murex brandaris), y ostión (Crassostrea angulata) con el $6 \%$ cada una de ellas y otras en menor cantidad como almejas y chirlas (Venerupis romboides, Tapes decussatus, Mactra carallina, Callista chione, Venus gallina), berdigones (Cerastoderma edule) o caracolas (Murex trunculus).

Al respecto de los crustáceos cabe decir que, para el mismo período y asentamientos antes referidos, el volumen de capturas fue de 46,26 toneladas correspondientes a trece especies, de las que la más abundante fue el centollo (Maia squinado) 19\%, cangrejo común o coñeta (Carcinus maenas) I5\%, langostino (Pnaeus kerathurus) 10\%, camarón de estero (Palaemonetes varians) y camarón rayado (Palaemon serratus) $8 \%$, boca (Uca tangeri), camarón duro (Sic-

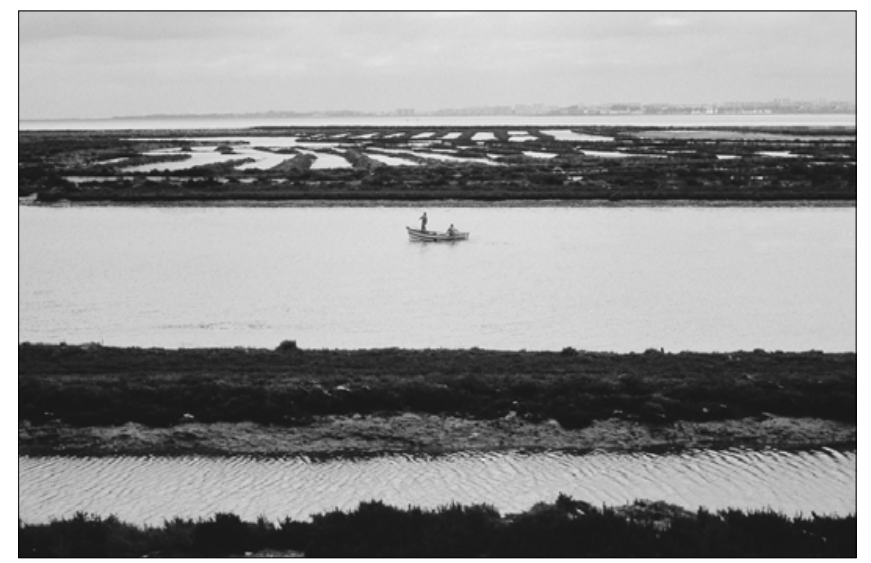

yonia carinata), cangrejo moro (Eriphia verrucosa) y cigalita (Upogebia deltadura) cada una con el $7 \%$ y en menor número la cigala (Nephrops norvegicus), galera (Squilla mantis) y santiaguiño (Scyllarus posteli). La mayoría de estas especies son capturadas para su consumo humano, pero algunas de ellas como la cigalita y algunos camarones son empleadas fundamentalmente como carnada para la pesca deportiva con caña.

Entre los equinodermos solo una especie es capturada y consumida en la Bahía, el erizo común (Paracentrotus lividus) que es capturado todo el año de forma manual en las zonas rocosas y constituye un plato típico de la cocina gaditana.

El de los poliquetos es otro grupo de especies objeto de un intenso volumen de capturas, siendo recolectado a pie y con herramientas manuales en los extensos fangales intermareales de la Bahía. Se utilizan exclusivamente para su uso como cebos de pesca alcanzando un alto valor comercial. De las cuatro especies capturadas, la gusana de canutillo (Diopatra napolitana) es la más numerosa con el $45 \%$, seguida de la miñoca (Nereis diversicolor) 30\%, gusana de sangre (Marpisa sanguinea) $20 \%$ y gusana de sebas (Arenicola marina) 5\%.

\section{La acuicultura: granjas marinas y parques de moluscos}

Aunque los denominados peces de estero eran ya apreciados desde que se pescaban en las salinas en que se criaban, y por tanto éstos en cierto modo podrían muy bien ser considerados como fruto de la acuicultura, realmente este aprovechamiento no tiene relevancia en la Bahía hasta que se producen la agudización de la crisis de la industria salinera gaditana y la reducción en el acceso a los caladeros de pesca internacionales.

Ya en los años cuarenta en plena crisis salinera algunas salinas modificaron en parte sus estructuras para permitir un incremento en el aprovechamiento secundario que hasta entonces obtenían de los peces que de forma natural se criaban y engordaban en su
La mayoría de las embarcaciones utilizadas en la Bahía para la pesca y el marisqueo son de pequeñas dimensiones y por lo general faenan de modo ilegal en zonas prohibidas.

Foto: Antón Ramírez 
La acuicultura intensiva implica una profunda transformación del medio para conseguir elevados rendimientos productivos.

Foto: Antón Ramírez

En el Parque Natural de la Bahía de Cádiz se conservan aun extensas playas en estado natural como esta de El Castillo en el municipio de San Fernando. Foto: F. J. Bravo
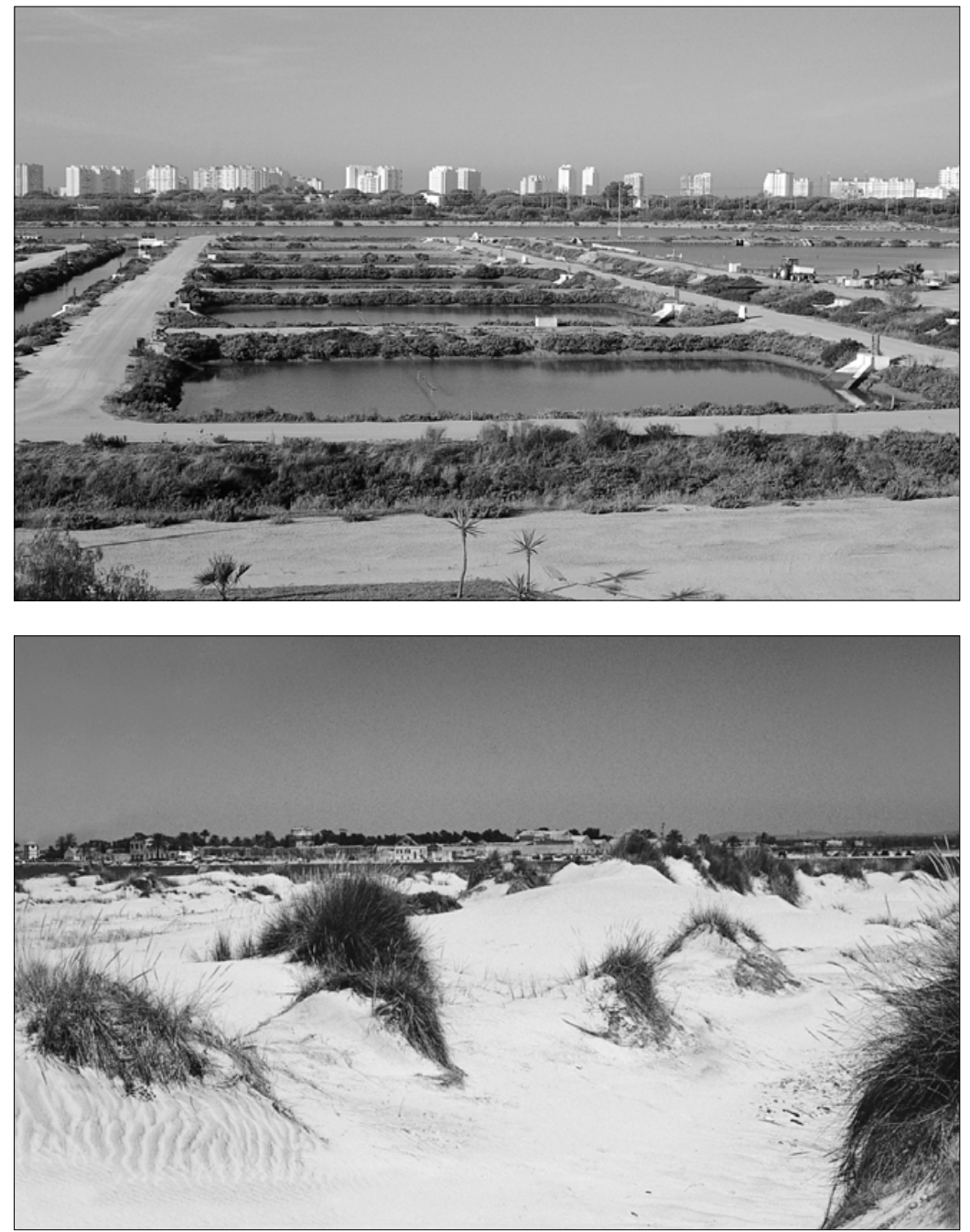
sea solo en el estero o en toda la superficie de la salina, que consiste en dejar engordar, sin suplemento alimenticio, a los alevines que de forma natural entran en la salina con el agua de los caños de alimentación, con ello se consigue un policultivo con una proporción alta de especies de escaso valor comercial como las lisas y baja de especies de mayor valor como la dorada cuyas producciones rondan solo los $5 \mathrm{Kg} / \mathrm{Ha} / a n ̃ o ~(4)$. Extensiva mejorada, se desarrolla interior, pero no es hasta el año 1980 cuando se inicia una intensiva reconversión del sector salinero hacia la acuicultura marina.

Actualmente existen aproximadamente unas 75 explotaciones dedicadas a la acuicultura en el Parque Natural de la Bahía de Cádiz y su entorno ocupando aproximadamente unas $3.500 \mathrm{Has}$. de la superficie de antiguas salinas (4). Su actividad se basa principalmente en la producción de doradas (Sparus aurata), en menor número lubinas (Dicentrarchus labrax) y lenguados (Solea senegalensis) y otras especies de menor valor comercial como las lisas y albures (Mugil cephalus, Liza spp., Chelon labrosus), anguilas (Anguilla anguilla) y bailas (Dicentrarchus punctatus).

La acuicultura en las salinas se desarrolla básicamente en tres modalidades (5): Extensiva tradicional, ya

\section{Los recursos naturales de valor estético y recreativo}

Hermosas playas y paisajes y una variada fauna, especialmente rica en aves, constituyen recursos naturales de elevado valor biológico, estético y recreativo en el ámbito del Parque Natural Bahía de Cádiz que, dado su gran potencial de atracción para el creciente ecoturismo, deben ser regulados en sus usos a fin de garantizar su conservación.

Magníficas playas, como la de Levante en el Puerto de Santa María y la de Camposoto o el Castillo en San Fernando, milagrosamente han llegado hasta nuestros días en un estado natural o seminatural en medio de un entorno profundamente transformado por la presión urbanística a que se ve sometida esta área altamente poblada. El uso intensivo y poco respetuoso con la naturaleza que puntualmente se hace de estos espacios debe ser regulado a corto plazo para evitar su creciente deterioro.

El saco interno de la Bahía, los caños y canales de sus marismas, presentan una gran belleza paisajística y son especialmente aptos para la práctica de actividades náuticas de carácter recreativo y deportivo por lo que constituyen per se otro valioso recurso natural que es aprovechado de forma intensiva por gran número de particulares y empresas turístico-recreativas dedicadas a estas actividades.

La gran riqueza y diversidad de aves acuáticas que están presentes en la Bahía gaditana durante todas las épocas del año la convierten en un lugar privile- 
giado en Europa para la observación de estas. Pueden verse aquí más de doscientas especies de aves y se llegan a alcanzar poblaciones que superan los 70.000 individuos en invernada estando especialmente bien representadas las limícolas, garzas, gaviotas, charranes y flamencos. Se trata igualmente de una zona fundamental para la reproducción de especies como la cigüeñuela, avoceta, chorlitejo patinegro, chararrancito o la espátula (7). El escaso conocimiento que de este hecho se tiene, tanto a nivel local como nacional e internacional, ha impedido hasta la fecha que en la Bahía se desarrolle el turismo ornitológico, sector en gran auge en toda Europa, que en un futuro inmediato, si se desarrollan las estrategias de divulgación necesarias para su despegue, podría tener un cierta relevancia en este Parque Natural.

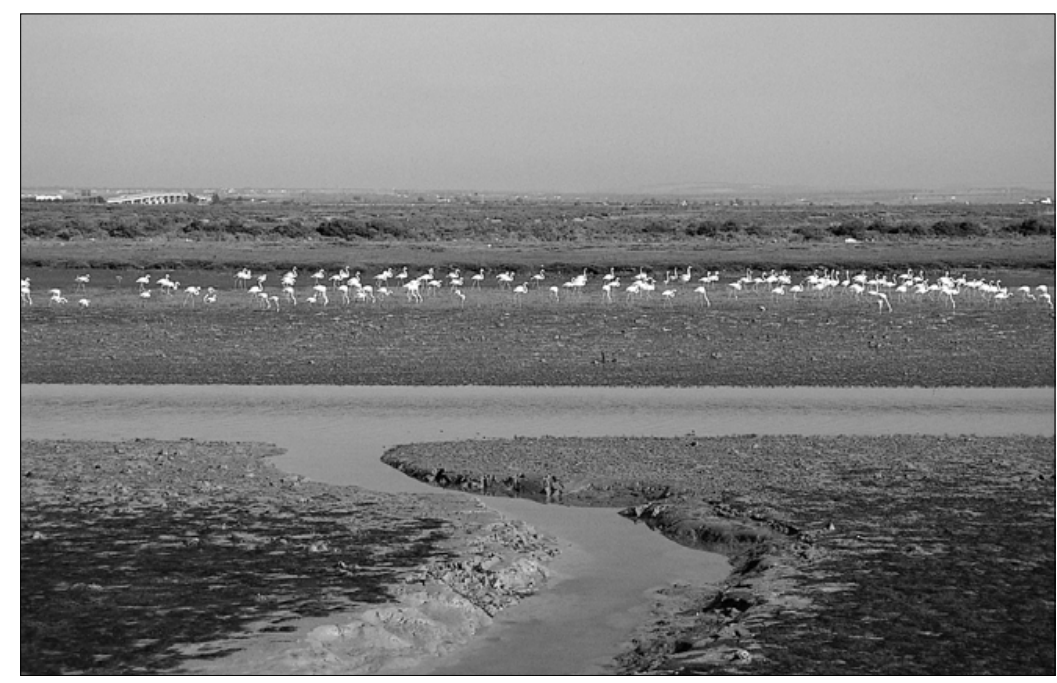

El turismo ornitológico podría constituir en un futuro próximo una importante fuente de recursos económicos en la Bahía.

Foto: F. J. Bravo

\section{Bibliografía}

(I) ARIAS, A.M.; DRAKE, P.: Estadios juveniles de la ictiofauna en los caños de la las salinas de la Bahía de Cádiz. Cádiz. Instituto de Ciencias Marinas de Andalucía (C.S.I.C) y Junta de Andalucía, 1990.

(2) ARIAS, A.M.; DRAKE, P.: Acuicultura en las salinas de la Bahía de Cádiz. En Acuicultura Marina. Fundamentos biológicos y tecnológicos de la producción. pp. 49-58. Barcelona. Universitat de Barcelona, 1993.

(3) ARIAS, A.M.; DRAKE, P.: Fauna acuática de las salinas del Parque Natural Bahía de Cádiz. Cádiz. Consejería de Medio Ambiente de la Junta de Andalucía, 1999.

(4) BARRAGÁN MUÑOZ, J. M. (coordinador): Estudios para la ordenación, planificación y gestión integrada de las zonas húmedas de la Bahía de Cádiz. Barcelona. Oikos-Tau, 1996.

(5) CONSEJERÍA DE MEDIO AMBIENTE DE LA JUNTA DE ANDALUCÍA: Guía del Parque Natural Bahía de Cádiz. Sevilla. Consejería de Medio Ambiente, 1998.

(6) CONSEJERÍA DE MEDIO AMBIENTE DE LA JUNTA DE ANDALUCÍA: Plan de Ordenación de los Recursos Naturales y Plan rector de Uso y Gestión del Parque Natural Bahía de Cádiz. Sevilla. Consejería de Medio Ambiente, 1997.
(7) GRUPO DE ESTUDIO DE AVES MARINAS Y LITORALES (GEAM): Cartografiado de las aves invernantes y reproductoras en el Parque Natural Bahía de Cádiz. Cádiz. Estudio del Departamento de Biología Animal, Vegetal y Ecología de la Universidad de Cádiz para la Consejería de Medio Ambiente de la Junta de Andalucía, 1997.

(8) GRUPO DE ESTUDIO DE AVES MARINAS Y LITORALES (GEAM): Bases para la regulación de la actividad marisquera en el Parque Natural de la Bahía de Cádiz. Cádiz. Estudio del Departamento de Biología Animal, Vegetal y Ecología de la Universidad de Cádiz para la Consejería de Medio Ambiente de la Junta de Andalucía, 2000.

(9) HERRERO LORENZO, M. P.: Estudio de las salinas de la bahía gaditana. Madrid. Tesis Doctoral del departamento de historia antigua de la Universidad Complutense de Madrid, 1978.

(10) VILLAR SERRANO, O.: La práctica de la pesca en la bahía interior de Cádiz y sus zonas contiguas de mar litoral. Cádiz. Tesis Doctoral del departamento de ciencias y técnicas de la navegación, máquinas y motores térmicos y teoría de la señal y comunicaciones de la Universidad de Cádiz, 1999. 